\title{
The Effect of Honokiol on Ergosterol Biosynthesis and Vacuole Function in Candida albicans
}

\author{
Lingmei Sun ${ }^{1 *}$ and Kai Liao ${ }^{2 *}$ \\ 'Department of Pharmacology, Medical School of Southeast University, Nanjing 210009, P.R. China \\ ${ }^{2}$ Department of Pathology and Pathophysiology, Medical School of Southeast University, Nanjing 210009, P.R. China
}

\begin{abstract}
Ergosterol, an essential constituent of membrane lipids of yeast, is distributed in both the cell membrane and intracellular endomembrane components such as vacuoles. Honokiol, a major polyphenol isolated from Magnolia officinalis, has been shown to inhibit the growth of Candida albicans. Here, we assessed the effect of honokiol on ergosterol biosynthesis and vacuole function in C. albicans. Honokiol could decrease the ergosterol content and upregulate the expression of genes related with the ergosterol biosynthesis pathway. The exogenous supply of ergosterol attenuated the toxicity of honokiol against C. albicans. Honokiol treatment could induce cytosolic acidification by blocking the activity of the plasma membrane Pma1 $\mathrm{p} \mathrm{H}^{+}$-ATPase. Furthermore, honokiol caused abnormalities in vacuole morphology and function. Concomitant ergosterol feeding to some extent restored the vacuolar morphology and the function of acidification in cells treated by honokiol. Honokiol also disrupted the intracellular calcium homeostasis. Amiodarone attenuated the antifungal effects of honokiol against $C$. albicans, probably due to the activation of the calcineurin signaling pathway which is involved in honokiol tolerance. In conclusion, this study demonstrated that honokiol could inhibit ergosterol biosynthesis and decrease Pma 1p $\mathrm{H}^{+}$-ATPase activity, which resulted in the abnormal $\mathrm{pH}$ in vacuole and cytosol.
\end{abstract}

Keywords: Candida albicans, honokiol, ergosterol biosynthesis, vacuole

Received: August 11, 2020 Accepted: November 24, 2020

First published online: November 27, 2020

*Corresponding authors L.Sun

Phone: +86-25-83272525 E-mail: sunlingmei@seu.edu.cn K.Liao

E-mail: liaokai0706@seu.edu.cn

Supplementary data for this paper are available on-line only at http://jmb.or.kr.

pISSN 1017-7825 elSSN 1738-8872

Copyright(C) 2020 by The Korean Society for Microbiology and Biotechnology

\section{Introduction}

Candida albicans, an opportunistic fungal pathogen in humans, is a common microbe residing on the oral mucosa, skin, and intestinal tract in healthy individuals $[1,2]$. It is worth noting that it may cause life-threatening invasive infections in immunocompromised patients and is listed as the fourth most common pathogenic microorganism of nosocomial bloodstream infections [2]. Several antifungal agents act by inhibiting the biosynthesis of ergosterol $[3,4]$. Both the reduced ergosterol and the excess of intermediate sterols are able to induce plasma membrane disorder and eventually lead to cell growth inhibition [5, 6]. Ergosterol, similar to cholesterol in mammals, is an essential constituent of membrane lipids. It modulates the fluidity, permeability, integrity, and thickness of the cell membrane [6]. A significant amount of ergosterol is found both in the cell membrane and in intracellular endomembrane components such as vacuoles $[7,8]$. Pmalp, which encodes the plasma membrane $\mathrm{H}^{+}$-ATPase, is the primary moderator of cytosolic $\mathrm{pH}$ in fungi. Its function is to extrude cytoplasmic hydrogen ion to maintain neutral-to-alkaline $\mathrm{pH}$ in the cytosol, and therefore, it is a potential drug target due to its necessity and specificity to fungi $[7,8]$.

The vacuole, analogous to the mammalian lysosome and the largest organelle in fungal cells, plays an essential role in a variety of cellular functions including cellular response to environmental stresses, ionic homeostasis, and the yeast-hyphae transition [9-11]. Vacuoles are needed to maintain intra-vacuolar $\mathrm{pH}$ and ion homeostasis, which is modulated by the vacuolar proton-translocating ATPase (V-ATPase) [12]. Cells show an abnormal morphology of the vacuole and a reduced degree of vacuolar acidification after treatment with ergosterol biosynthesis inhibitors such as azoles or morpholines [8]. V-ATPase is found in all eukaryotic cells and is responsible for the acidification of intracellular compartments by driving the translocation of protons across the vacuolar membrane into the lumen $[8,12]$. Therefore, V-ATPase is critical for maintaining cellular ion homeostasis.

Magnolia officinalis, a plant with a long history of use in traditional Asian medicine, is administered clinically to treat bacterial infections, inflammation, and gastrointestinal diseases [13-15]. Honokiol, a bioactive polyphenol, is one of the main active ingredients in M. officinalis. Honokiol has been found to induce reactive oxygen species accumulation through mitochondrial dysfunction in C. albicans $[16,17]$. Recently, it has been shown that altered sterol metabolism could decrease Fe-S cluster synthesis, and thus decrease mitochondrial function [18]. To 
further reveal the underlying mechanism of honokiol against C. albicans, we studied the effect of honokiol on ergosterol biosynthesis and vacuole function in C. albicans.

\section{Materials and Methods}

\section{Materials}

Honokiol (5,5'-diallyl-2,4'-dihydroxybiphenyl) was obtained from Xi'an Yuquan Biological Technology Co., Ltd. and its purity was over $98 \%$ as analyzed by high-performance liquid chromatography. Yeast vacuole membrane marker MDY-64 was purchased from Invitrogen (Thermo Fisher Scientific, USA). EGTA, ergosterol, and amiodarone (AMD) were purchased from Aladdin Bio-Chem Technology Co., Ltd. (China). For analysis of exogenous sterol utilization, ergosterol was dissolved in a mixture of ethanol (50\%) and Tween-80 (50\%) to give a $10 \mathrm{mM}$ stock solution, which was used to supplement the liquid medium with a final concentration of $50 \mu \mathrm{M}$. The same concentration of vehicle without ergosterol was used as a control.

\section{Strains and Media}

C. albicans strain SC5314 was stored with $15 \%$ glycerol as frozen stock at $-80^{\circ} \mathrm{C}$. Before each experiment, cells were freshly revived on YPD (yeast extract/ peptone/dextrose) agar plate from the stock.

\section{Ergosterol Quantification}

An overnight liquid culture of C. albicans SC5314 was diluted 100-fold with YPD medium and exposed to drug treatment. After $12 \mathrm{~h}$ incubation, the cells were harvested by centrifugation. Cell total sterol was extracted and measured as previously described [19].

\section{Quantitative RT (qRT)-PCR Assay}

C. albicnas strain SC5314 was grown overnight in YPD medium. Yeasts cells which were resuspended at a cell density of $1.0 \times 10^{7}$ cells $/ \mathrm{ml}$ were treated with $16 \mu \mathrm{g} / \mathrm{ml}$ honokiol for $12 \mathrm{~h}$ at $30^{\circ} \mathrm{C}$. Total RNA was extracted by the hot phenol method [19]. About $1 \mu \mathrm{g}$ of RNA was reverse-transcripted into cDNA using AMV reverse transcriptase (Promega, USA). Primer sequences of genes are shown in supplementary Table S1. The qRT-PCR and data analysis were conducted as previously described $[16,17]$.

\section{Measurement of Acidification of the External Medium}

The P-type $\mathrm{H}^{+}$-ATPase has been proved to relate with ergosterol-enriched domains [7, 8]. It pumps protons out of cells to acidify the external environment as soon as glucose is activated $[7,8]$. To estimate the effect of honokiol on the function of Pma1, we tested acidification of the extracellular medium via glucose activation in honokioltreated yeast cells. The effect of honokiol on glucose-induced acidification of the external medium was tested according to the method as previously described [20]

\section{Isolation of Plasma Membrane and Measurement of ATP Hydrolysis}

Isolation of plasma membrane was performed as described previously [20]. The ATP hydrolysis method was tested in a medium $\left(0.2 \mathrm{mM}\right.$ EDTA, $60 \mathrm{mM}$ Tris, $8 \mathrm{mM} \mathrm{MgCl}_{2}, \mathrm{pH}$ 5.7). After incubating the plasma membrane at $37^{\circ} \mathrm{C}$ for $10 \mathrm{~min}$ with or without honokiol, the reaction was triggered by the addition of ATP $(50 \mathrm{mM})$ and then incubated for $10 \mathrm{~min}$. Cold trichloroacetic acid (5\%) was added to stop the progress of the reaction. Free inorganic phosphate was analyzed as described previously [20].

\section{Vacuole Staining and Vacuolar pH Measurements}

MDY-64, a yeast vacuole membrane marker, was used to visualize vacuoles according to the manual description. Vacuole morphology was monitored by fluorescence microscopy using an FITC filter set (Olympus IX71, Olympus Co., Japan). Quinacrine, which shows green fluorescence in acidic condition, was employed to estimate the vacuolar $\mathrm{pH}$ in living C. albicans cells qualitatively [21]. Yeast cells were exposed to drugs for $4 \mathrm{~h}$, and then the cells were stained with $200 \mu \mathrm{M}$ quinacrine. The fluorescence images of cells were obtained by the fluorescence microscope using the FITC filter set.

\section{Measurement of the Relative Levels of Intracellular Free $\mathrm{Ca}^{2+}$}

The relative levels of intracellular free $\mathrm{Ca}^{2+}$ were determined by the $\mathrm{Ca}^{2+}$-sensitive indicator Fura 2-AM. C. albicans strain was cultured overnight at $30^{\circ} \mathrm{C}$ in YPD medium and washed with PBS buffer, and $1 \times 10^{7}$ cells were resuspended in PBS (without $\mathrm{CaCl}_{2}$ and $\mathrm{MgCl}_{2}$ ) plus $10 \mu \mathrm{M}$ Fura 2-AM at $37^{\circ} \mathrm{C}$ for $1 \mathrm{~h}$. After washing three times with PBS, Fura-2 fluorescence was determined by excitation wavelength at $340 \mathrm{~nm}$ and $380 \mathrm{~nm}$, and emission wavelength at $510 \mathrm{~nm}$ in a BioTek Synergy 4 microplate reader (BioTek Instruments, Inc.), with relative levels of intracellular $\mathrm{Ca}^{2+}$ being expressed as the ratio of the fluorescence intensity upon excitation at $340 \mathrm{~nm}$ $\left(\mathrm{F}_{340}\right)$ to the $\mathrm{F}_{380}$.

\section{Results}

\section{Honokiol Inhibits Ergosterol Biosynthesis}

Ergosterol is an essential component of yeast cell membrane lipids [6]. Because of its absence in mammals, targeting ergosterol is appealing and effective as a therapeutic avenue $[3,5]$. To verify if honokiol could inhibit ergosterol biosynthesis, we measured the cellular ergosterol level using a spectrophotometric assay. As expected, 
A

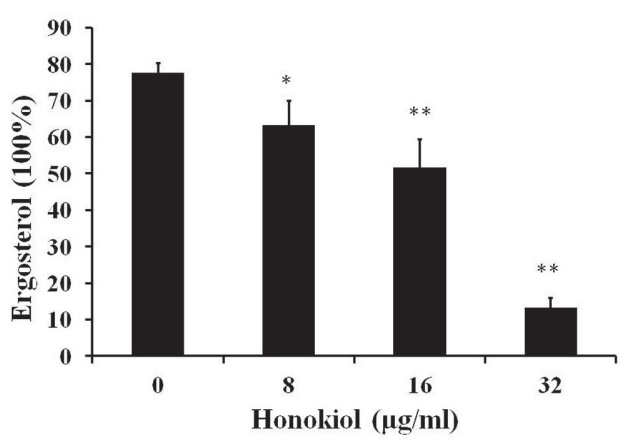

B

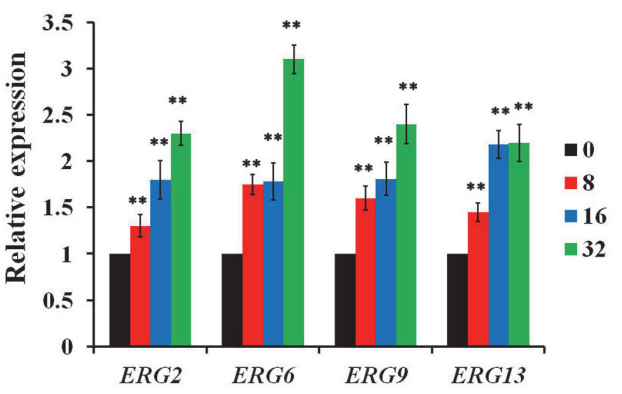

C

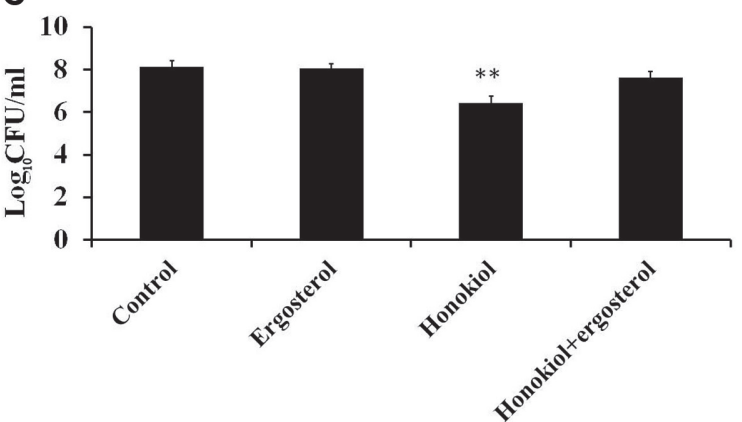

Fig. 1. The effect of honokiol on ergosterol biosynthesis. (A) Honokiol decreased the ergosterol level in C. albicans. Ergosterol was extracted from cells treated by different concentrations of honokiol and measured by the spectrophotometric method. (B) qRT-PCR of the genes involved in ergosterol biosynthesis. C. albicans was treated with different concentrations of honokiol for $12 \mathrm{~h}$. (C) The effect of exogenous ergosterol on the antifungal activity of honokiol. C. albicans was treated with honokiol $(16 \mu \mathrm{g} / \mathrm{ml})$, ergosterol $(50 \mu \mathrm{M})$ or their combination. ${ }^{*} p<0.05 ;{ }^{* *} p<0.01$.

the ergosterol content significantly decreased in the cells treated with honokiol compared with the control group (Fig. 1A). To further investigate the effect of honokiol on the expression of ergosterol biosynthesis genes, real-time RT-PCR analysis was employed. The results showed the upregulation of ergosterol synthesis genes in response to honokiol treatment (Fig. 1B). Also, feeding exogenous ergosterol could reverse the inhibition of cell growth caused by honokiol treatment (Fig. 1C). The ability of exogenous ergosterol to restore the cell growth after honokiol treatment supports a hypothesis that antifungal activity of honokiol is partly a result of the ergosterol depletion.

\section{Honokiol Disrupts $\mathrm{H}^{+}$-ATPase Activity}

The plasma membrane $\mathrm{H}^{+}$-ATPase Pmal, the primary modulator of cytosolic $\mathrm{pH}$ in fungi, has been demonstrated to associate with ergosterol-enriched domains $[7,9]$. To estimate the effect of honokiol on Pmal function, we measured the acidification of the extracellular medium upon glucose activation in honokiol treatment cells. As shown in Fig. 2A, the acidification curve of the extracellular medium was revealed to be quite different between the control and honokiol-treated group. Honokiol treatment slowed the medium acidification rate relative to wild type, as shown in Fig. 2A. Differences between control and honokiol-treated group in acidification of their external environments indicate a significantly reduced $\mathrm{H}^{+}$-ATPase activity in the strain with honokiol treatment compared to the control group. We isolated the plasma membranes and detected the ATPasespecific activity. Treatment by honokiol led to decrease in $\mathrm{H}^{+}$-ATPase activity in the plasma membrane in a concentration-dependent manner (Fig. 2B). We further measured the expression of the PMA1 gene. As expected, honokiol treatment resulted in a lower level of PMA1 gene expression than in the control group (Fig. 2C). This low level of PMA1 gene expression could also result in a low level of Pmalp. Together these findings suggest that honokiol could decrease the amount of Pmalp in the plasma membrane as well as the activity of the Pmalp enzyme itself.

\section{Honokiol Affects Vacuole Morphology and Acidification}

To investigate the effect of honokiol on the vacuole, we analyzed the vacuole structure and function after treatment by honokiol. We used the yeast vacuole marker MDY-64 to stain yeast vacuolar membranes. As shown in Fig. 3A, the vehicle control strains exhibited the typical ring-staining pattern of the vacuole membrane. In contrast, yeast vacuole marker MDY-64 diffusely distributed in the cytoplasm in the honokiol-treated strains. This result suggested honokiol induced abnormal vacuole morphology. We also assessed the effect of the exogenous supply of ergosterol on vacuole morphology treatment by honokiol. As shown in Fig. 3A, compared 
A
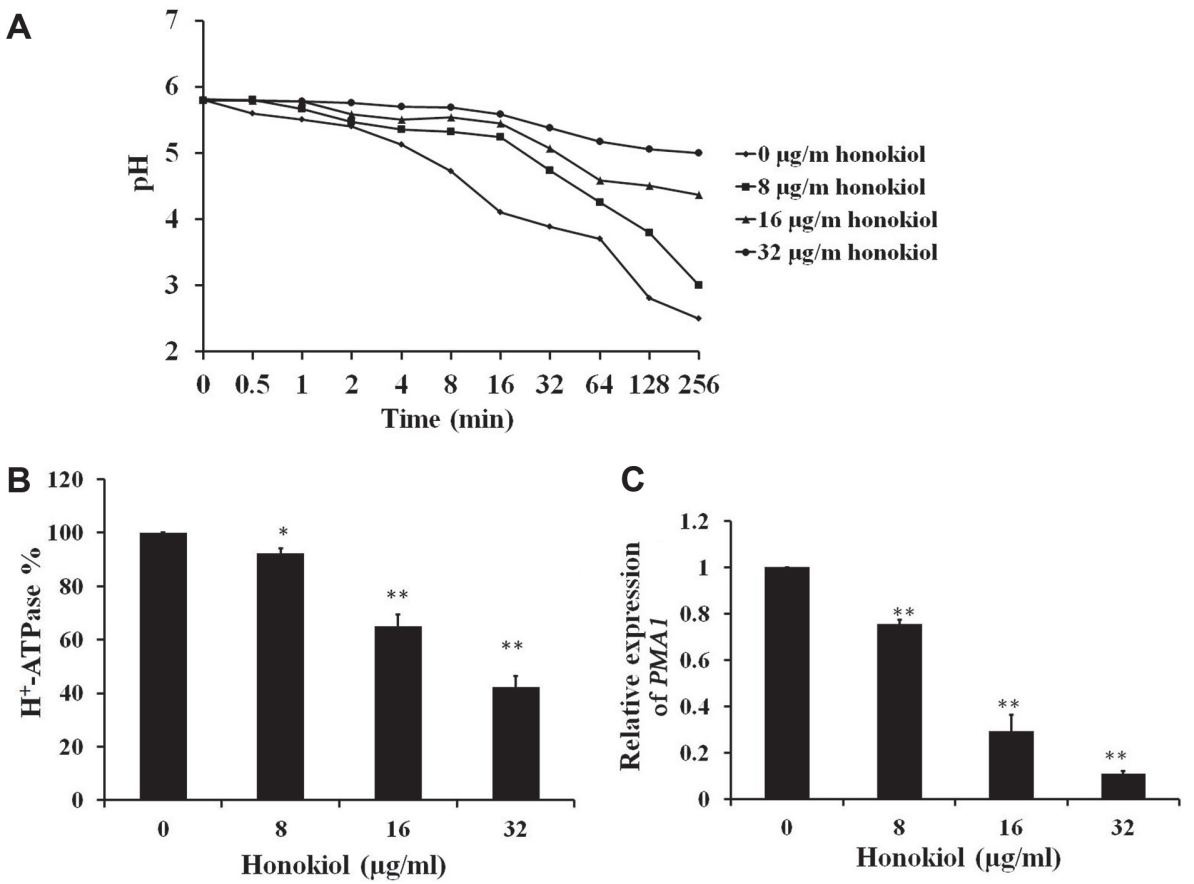

Fig. 2. Honokiol impaired the function of Pmalp. (A) The inhibitory effect of honokiol on the glucose-dependent acidification of medium in C. albicans. Cells were diluted to $3 \sim 5 \times 10^{7} \mathrm{CFU} / \mathrm{ml}$. Extracellular $\mathrm{pH}$ was recorded after glucose was added to $2 \%$ at time 0 . A representative of several experiments with very similar results is shown. (B) Honokiol decreased plasma membrane ATPase activity. The percentage of ATPase-specific activity in isolated plasma membranes treatment by different concentrations of honokiol as compared to ATPase-specific activity in the control was calculated. (C) The effect of honokiol on the expression of the PMA1 gene. C. albicans was treated with different concentrations of honokiol for $12 \mathrm{~h}$. Bars represent mean $\pm \mathrm{SD}$. ${ }^{*} p<0.05 ;{ }^{* *} p<0.01$.

A

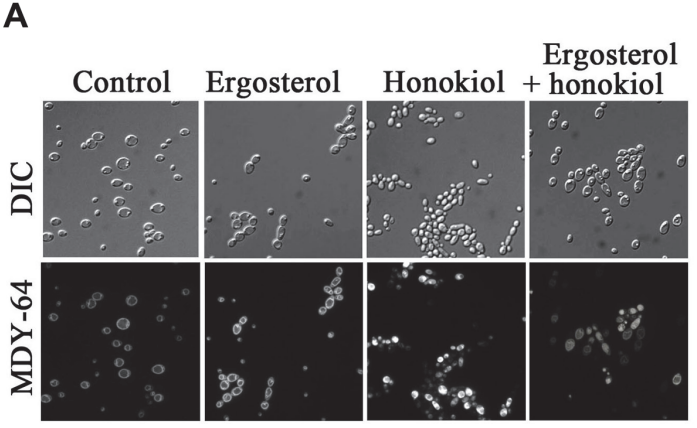

B

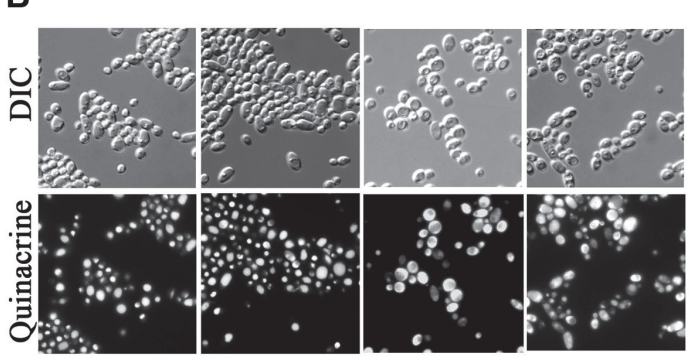

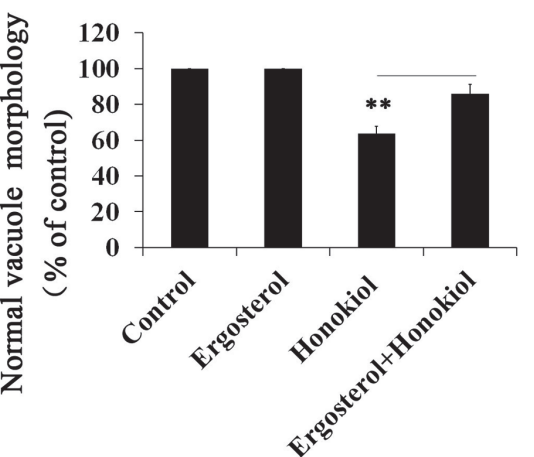

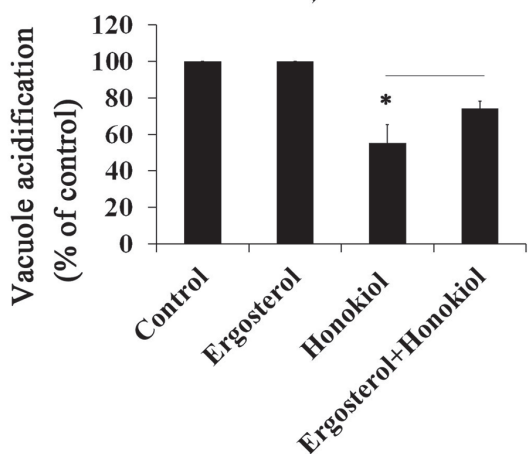

Fig. 3. The effect of honokiol on the vacuole morphology and acidification in C. albicans. (A) Vacuole morphology was observed using the fluorescein stain yeast vacuole marker MDY-64. (B) Vacuole acidification was assessed by quinacrine which is accumulated in the acidic compartment. C. albicans was treated with honokiol $(16 \mu \mathrm{g} / \mathrm{ml})$, ergosterol $(50 \mu \mathrm{M})$ or their combination. Bars represent mean \pm SD. ${ }^{*} p<0.05 ;{ }^{* *} p<0.01$. 

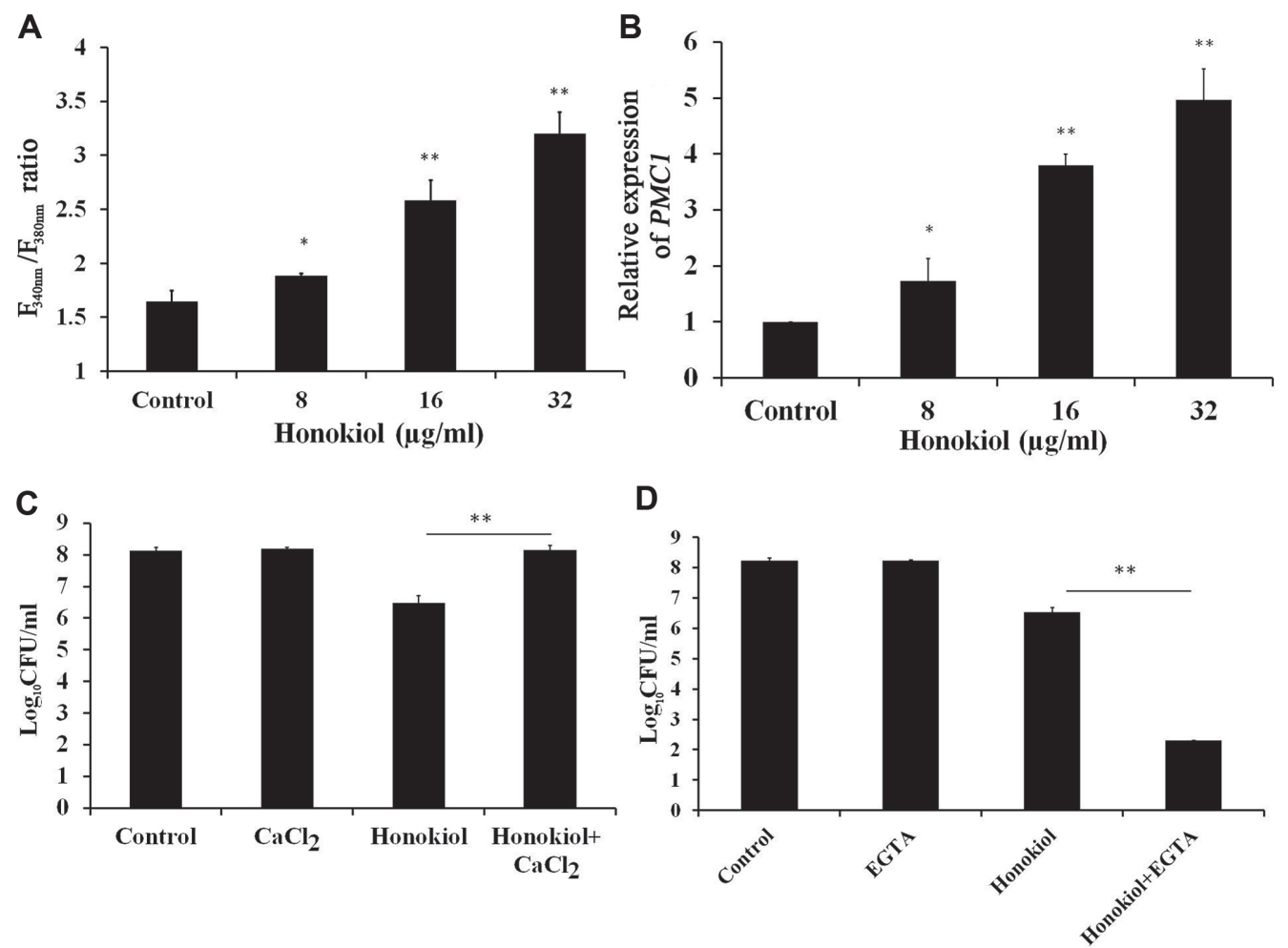

Fig. 4. The intracellular calcium level was related with honokiol tolerance against C. albicans. (A) Honokiol treatment induced abnormal intracellular calcium levels. Cells were grown in YPD liquid medium at $30^{\circ} \mathrm{C}$, labeled with calcium-responsive dye Fura 2-AM. The fluorescence intensity was tested with excitation at $340 \mathrm{~nm}$ or $380 \mathrm{~nm}$ and emission at $510 \mathrm{~nm}$. Intracellular calcium levels are expressed as $\mathrm{F}_{340} / \mathrm{F}_{380}$ ratios. (B) The effect of honokiol on the expression of $P M C 1$ gene. C. albicans was treated with different concentrations of honokiol for $12 \mathrm{~h}$. (C and D) The effect of exogenous calcium (C) or calcium chelator EGTA (D) on the antifungal activity of honokiol. C. albicans was treated with honokiol $(16 \mu \mathrm{g} / \mathrm{ml})$, calcium $(10 \mathrm{mM}) / \mathrm{EGTA}(10 \mathrm{mM})$ or their combination. Bars represent mean $\pm \mathrm{SD} .{ }^{*} p<0.05 ;{ }^{* *} p<0.01$.

with cells treated with honokiol alone, ergosterol decreased the abnormal vacuole morphology to some degree.

A variety of methods can be used to assess the relative acidification of the vacuole [22]. Quinacrine, a weak base, is frequently used to label the vacuole [21]. Because of its lipophilic nature, quinacrine is able to diffuse across the vacuolar membrane. Still, once exposed to the low $\mathrm{pH}$ of the vacuolar lumen it becomes protonated and is unable to leave the organelle [22]. In the vehicle control group, the vacuole was clearly labeled with quinacrine. However, under honokiol treatment, quinacrine fluorescence did not colocalize within the vacuole lumen, but was diffusely distributed in the cytoplasm (Fig. 3B), and therefore appears to be defective in vacuolar acidification. The impaired trafficking of quinacrine to the vacuolar lumen in the honokiol-treated strains may be caused by cytosolic acidification and vacuolar alkalization. Moreover, the exogenous ergosterol could recover the localization of quinacrine to some extent (Fig. 3B).

\section{Honokiol Disrupts Intracellular Calcium Homeostasis}

In the fungal cell, more than $90 \%$ of the intracellular $\mathrm{Ca}^{2+}$ is stored in the vacuole. Maintenance of calcium homeostasis is essential for yeast cells to resist environmental stresses such as drug exposure. Hence, to observe whether the vacuolar trafficking defects by honokiol impact cellular $\mathrm{Ca}^{2+}$ homeostasis, the calcium-sensitive dye Fura 2-AM was employed to detect the relative levels of intracellular free calcium. As shown in Fig. $4 \mathrm{~A}$, the $\mathrm{F}_{340} / \mathrm{F}_{380}$ ratio of Fura 2-AM-stained honokiol-treated cells was significantly higher than that of the vehicle-treated group, suggesting that honokiol-treated cells have abnormally high intracellular $\mathrm{Ca}^{2+}$ levels (Fig. 4A). Pmc1, the yeast $\mathrm{Ca}^{2+}$ pump localizes to the vacuolar membrane $\mathrm{Ca}^{2+}$-ATPase, is induced under calcium stress and serves to detoxify excess $\mathrm{Ca}^{2+}$ by sequestration into the vacuole [23]. Fluconazole treatment could strongly induce the expression of $P M C 1$ gene, a downstream effector of calcineurin signaling $[23,24]$. In our study, $P M C 1$ expression was also enhanced approximately fourfold in cells exposed to honokiol $(16 \mu \mathrm{g} / \mathrm{ml})$ compared with control cells (Fig. 4B). The possibility was raised that $P M C 1$ could play a survival role in the presence of honokiol. Also, the exogenous calcium addition in the medium which could activate calcineurin enhanced the tolerance to honokiol (Fig. 4C). When we used the $\mathrm{Ca}^{2+}$ chelator EGTA to bind free $\mathrm{Ca}^{2+}$ in the medium, the supplement of $10 \mathrm{mM}$ EGTA increased the susceptibility of honokiol against C. albicans (Fig. 4D). Taken together, our results support the increase of honokiol-induced intracellular $\mathrm{Ca}^{2+}$ levels as a protective effect against honokiol-induced cell death. 


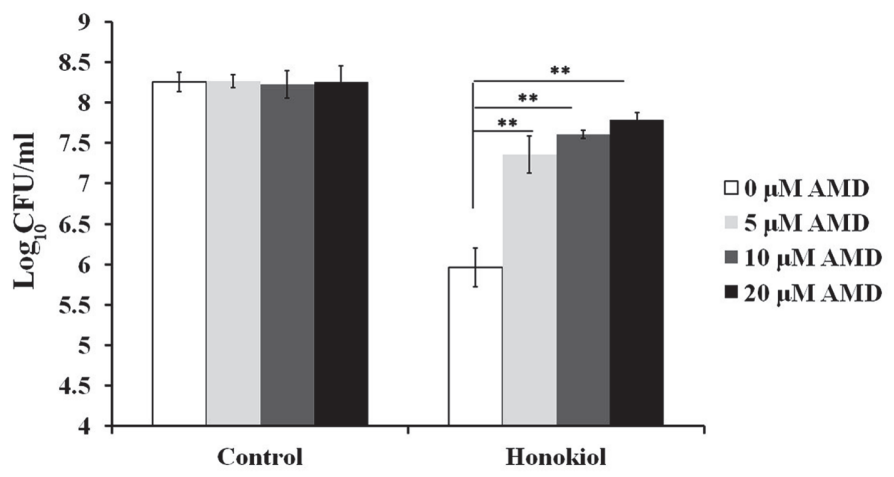

Fig. 5. The effect of AMD on the antifungal activity of honokiol. C. albicans was treated with honokiol $(16 \mu \mathrm{g} / \mathrm{ml})$, $\operatorname{AMD}(5,10,20 \mu \mathrm{M})$ or their combination. Bars represent mean \pm SD. ${ }^{*} p<0.01$.

\section{In Vitro Interactions between Honokiol and AMD}

AMD, an antiarrhythmic drug, has a prominent and rapid effect on C. albicans, which is most noticeably reflected as changes in calcium stress pathways [25]. It could elicit an immediate influx of calcium and the increased the expression of calcineurin-regulated $\mathrm{C} 2 \mathrm{H} 2$ transcription factor CRZ1 [8, 25]. To assess the effect of AMD on the anti-candidal activity of honokiol, we added various amounts of AMD to medium with or without honokiol. As shown in Fig. 5, culture containing AMD and honokiol combinations were found to have a significant increase in CFU relative to culture without AMD. This result suggests that AMD-induced calcium influx could alleviate honokiol toxicity.

\section{Discussion}

As a primary constituent of cell membranes, the depletion of ergosterol alters many intracellular biological reactions and changes membrane properties such as fluidity, permeability, and thickness [3,4]. Ergosterol also has essential roles in mitochondria, vacuoles, and lipid rafts in C. albicans [8]. Due to the absence of ergosterol in mammals, ergosterol as a target of antifungal drugs has excellent advantages [8,26, 27]. In our study, honokiol could decrease the ergosterol content and disturb the expression of genes related to ergosterol biosynthesis (Figs. 1A and 1B). The upregulation of global ERG genes was also observed following exposure to ergosterol biosynthesis inhibitors in C. albicans $[18,28,29]$. However, the molecular mechanism behind this compensatory response pathway is still mostly unknown. Furthermore, concomitant addition of ergosterol restored the cell growth after honokiol treatment in C. albicans (Fig. 1C). Thus, depletion of ergosterol is a plausible mechanism for the antifungal activity of honokiol.

The P-type $\mathrm{H}^{+}$-ATPase Pma1 related to ergosterol-enriched domains is the primary regulator of cytosolic $\mathrm{pH}$ in fungi $[7,8]$. Upon glucose activation, it extrudes protons out of cells to maintain normal intracellular $\mathrm{pH}$ and acidifies the extracellular medium $[30,31]$. The lack of ergosterol in the plasma membrane, as well as a reduced level of ATP after honokiol treatment as demonstrated previously in C. albicans may cause the abnormal functioning of $\mathrm{H}^{+}$-ATPase (Fig. 2). We also observed the lower expression levels of the PMA1 gene in C. albicans treatment by honokiol (Fig. 2C). Honokiol blocks Pmalp $\mathrm{H}^{+}$-ATPase by inhibiting the transport of cytosolic protons out of the cell. Given all these results, we reasoned that honokiol treatment could induce cytosolic acidification by blocking the activity of Pmalp $\mathrm{H}^{+}$-ATPase.

The vacuole, the largest organelle in yeast cells, plays an essential role in multiple intracellular functions such as the response to environmental conditions and the yeast-to-hyphae transition [8-10]. Vacuoles are needed to maintain the intravacuolar homeostasis of $\mathrm{pH}$ and ion [32]. In our study, we used two fluorescent dyes, the yeast vacuole membrane marker MDY-64 and the weak base quinacrine, to observe the effect of honokiol on the morphology of the vacuole and vacuolar acidification, respectively (Figs. 3A and 3B). We observed that honokiol induced the abnormal morphology and defective acidification of the vacuole (Fig. 3B). There are previously reported data that cells with ERG2 or ERG24 deletion, or exposure to ergosterol biosynthesis inhibitors morpholines or azoles, decrease the degree of vacuolar acidification and show an abnormal morphology of the vacuole [33]. On this account, we infer that honokiol induced the damage to vacuole morphology and function partly due to ergosterol depletion. A previous study suggested a role for sphingolipids in helping to maintain vacuole morphology and function [34]. Myriocin, a metabolite isolated from the insect fungus Isaria sinclairii, irreversibly inhibits serine palmitoyltransferase, and thus decreases the sphingolipid synthesis [35]. Both ergosterol and sphingolipid depletion could induce abnormal vacuole morphology and function (Fig. S1). Furthermore, increased levels of cytosolic calcium correlate with vacuolar fragmentation [36]. Further studies are needed to research the mechanism of honokiol on abnormal vacuolar morphology and function.

Maintenance of calcium homeostasis is essential for cells to respond to various stresses [37]. The vacuole is a major calcium store in many organisms, particularly plants and fungi [23]. Upon appropriate stimulation, calcium is released rapidly from extracellular sources as well as intracellular stores into the cytoplasm to activate calciumdependent protein molecules such as calcineurin $[25,38]$. In this study, honokiol treatment induced the accumulation of calcium and the upregulation of PMC1 (Figs. 4A and 4B). Upon the extra calcium accumulation, 


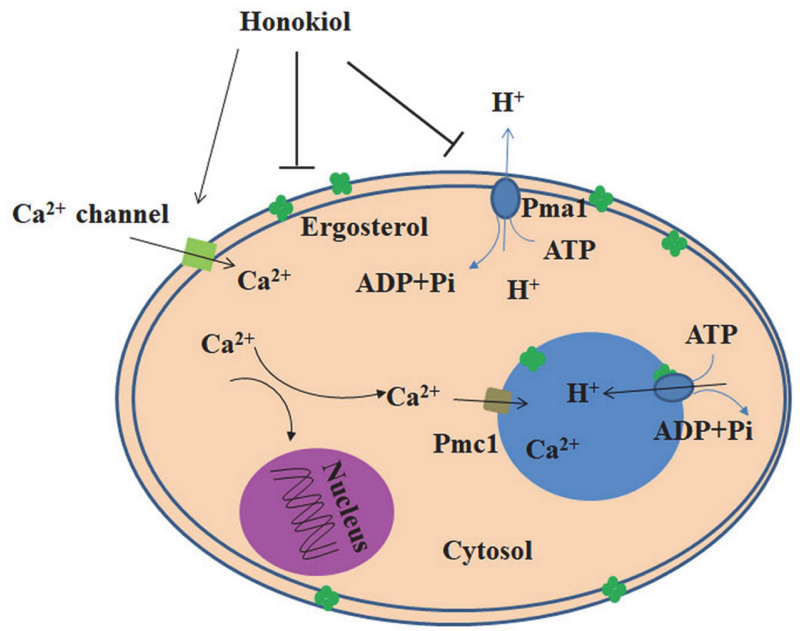

Fig. 6. A hypothetical model for the effect of honokiol on ergosterol biosynthesis and vacuole function in C. albicans.

Pmclp localizing to the vacuolar membrane could trap excess calcium into the vacuole. Also, the increase in calcium accumulation could lead to activate the calcineurin signaling pathway [23]. The exogenous calcium weakened the toxicity of honokiol against $C$. albicans (Fig. 4C). By contrast, calcium chelator EGTA increased the susceptibility of honokiol against C. albicans (Fig. 4D). This is probably due to the activation of the calcineurin signaling pathway being involved in honokiol tolerance against C. albicans.

In Saccharomyces cerevisiae, AMD could increase $\mathrm{Ca}^{2+}$ influx, so it affects calcium homeostasis, leading to the activation of the calcineurin pathway $[8,25]$. A previous study provided in vitro evidence that AMD is obviously synergistic with azoles against $C$. albicans [39]. On the contrary, in our study, AMD attenuated the antifungal activity of honokiol against C. albicans (Fig. 5). Due to the AMD and honokiol combination yielding a mean increase of $\log _{10} \mathrm{CFU} / \mathrm{ml}$ less than $2 \log _{10} \mathrm{CFU} / \mathrm{ml}$ compared with honokiol treatment alone, the interaction of $\mathrm{AMD}$ and honokiol was indifferent. This indifferent interaction was also determined using the checkerboard method (data not shown).

Overall, the present study supports evidence that honokiol inhibits ergosterol biosynthesis and decreases Pmal $\mathrm{H}^{+}$-ATPase activity, resulting in abnormal $\mathrm{pH}$ in the vacuole and cytosol (Fig. 6). The increased calcium level has been shown to induce adaptive responses that assist $C$. albicans survival following honokiol treatment. The results of this study will contribute to a better understanding of the antifungal mechanism of honokiol.

\section{Acknowledgments}

This work was supported by grants from National Natural Science Foundation of China (81703574), Jiangsu Province Natural Science Foundation (BK20140624), and the Fundamental Research Funds for the Central Universities.

\section{Conflict of Interest}

The authors have no financial conflicts of interest to declare.

\section{References}

1. Rodrigues CF, Rodrigues ME, Henriques M. 2019. Candida sp. Infections in patients with diabetes mellitus. J. Clin. Med. 8: 76.

2. Da SDA, Lee KK, Raziunaite I, Schaefer K, Wagener J, Yadav B, et al. 2016. Cell biology of Candida albicans-host interactions. Curr. Opin. Microbiol. 34: 111-118.

3. Villasmil ML, Barbosa AD, Cunningham JL, Siniossoglou S, Nickels JJ. 2020. An Erg11 lanosterol 14-alpha-demethylase-Arv1 complex is required for Candida albicans virulence. PLoS One 15: e235746.

4. Kean R, Ramage G. 2019. Combined antifungal resistance and biofilm tolerance: the global threat of Candida auris. mSphere 4: e00458-19.

5. Sanguinetti M, Posteraro B, Fiori B, Ranno S, Torelli R, Fadda G. 2005. Mechanisms of azole resistance in clinical isolates of Candida glabrata collected during a hospital survey of antifungal resistance. Antimicrob. Agents Chemother. 49: 668-679.

6. Gao J, Wang H, Li Z, Wong AH, Wang YZ, Guo Y, et al. 2018. Candida albicans gains azole resistance by altering sphingolipid composition. Nat. Commun. 9: 4495.

7. Suchodolski J, Muraszko J, Bernat P, Krasowska A. 2019. A crucial role for ergosterol in plasma membrane composition, localisation, and activity of Cdr1p and $\mathrm{H}^{+}$-ATPase in Candida albicans. Microorganisms 7: 378.

8. Zhang YQ, Gamarra S, Garcia-Effron G, Park S, Perlin DS, Rao R. 2010. Requirement for ergosterol in V-ATPase function underlies antifungal activity of azole drugs. PLoS Pathog. 6: e1000939.

9. Martínez-Muñoz GA, Kane P. 2008. Vacuolar and plasma membrane proton pumps collaborate to achieve cytosolic pH homeostasis in yeast. J. Biol. Chem. 283: 20309-20319.

10. Minematsu A, Miyazaki T, Shimamura S, Nishikawa H, Nakayama H, Takazono T, et al. 2019. Vacuolar proton-translocating ATPase is required for antifungal resistance and virulence of Candida glabrata. PLoS One 14: e210883. 
11. Merz AJ, Wickner WT. 2004. Trans-SNARE interactions elicit $\mathrm{Ca}^{2+}$ efflux from the yeast vacuole lumen. J. Cell Biol. 164: 195-206.

12. Hurst LR, Fratti RA. 2020. Lipid rafts, sphingolipids, and ergosterol in yeast vacuole fusion and maturation. Front. Cell Dev. Biol. 8: 539 .

13. Li Y, Xu C, Zhang Q, Liu JY, Tan RX. 2005. In vitro anti-Helicobacter pylori action of 30 Chinese herbal medicines used to treat ulcer diseases. J. Ethnopharmacol. 98: 329-333.

14. Liao K, Sun L. 2018. Roles of the Hsp90-calcineurin pathway in the antifungal activity of honokiol. J. Microbiol. Biotechnol. 28: 10861093.

15. Sun L, Ye X, Ding D, Kai L. 2019. Opposite effects of vitamin C and vitamin E on the antifungal activity of honokiol. J. Microbiol. Biotechnol. 29: 538-547.

16. Sun L, Liao K, Hang C, Wang D. 2017. Honokiol induces reactive oxygen species-mediated apoptosis in Candida albicans through mitochondrial dysfunction. PLoS One 12: e172228.

17. Sun L, Liao K, Wang D. 2017. Honokiol induces superoxide production by targeting mitochondrial respiratory chain complex I in Candida albicans. PLoS One 12: e184003.

18. Ward DM, Chen OP, Li L, Kaplan J, Bhuiyan SA, Natarajan SK, et al. 2018. Altered sterol metabolism in budding yeast affects mitochondrial iron-sulfur (Fe-S) cluster synthesis. J. Biol. Chem. 293: 10782-10795.

19. Sun LM, Liao K, Liang S, Yu PH, Wang DY. 2015. Synergistic activity of magnolol with azoles and its possible antifungal mechanism against Candida albicans. J. Appl. Microbiol. 118: 826-838.

20. Wu XZ, Cheng AX, Sun LM, Sun SJ, Lou HX. 2009. Plagiochin E, an antifungal bis(bibenzyl), exerts its antifungal activity through mitochondrial dysfunction-induced reactive oxygen species accumulation in Candida albicans. Biochim. Biophys. Acta 1790: 770-777.

21. Kulkarny VV, Chavez-Dozal A, Rane HS, Jahng M, Bernardo SM, Parra KJ, et al. 2014. Quinacrine inhibits Candida albicans growth and filamentation at neutral pH. Antimicrob. Agents Chemother. 58: 7501-7509.

22. Perzov N, Padler-Karavani V, Nelson H, Nelson N. 2002. Characterization of yeast V-ATPase mutants lacking Vph1p or Stv1p and the effect on endocytosis. J. Exp. Biol. 205: 1209-1219.

23. Luna-Tapia A, DeJarnette C, Sansevere E, Reitler P, Butts A, Hevener KE, et al. 2019. The vacuolar Ca ${ }^{2+}$ ATPase pump pmclp is required for Candida albicans pathogenesis. $m$ Sphere 4: e00715-18.

24. Chen YL, Brand A, Morrison EL, Silao FG, Bigol UG, Malbas FJ, et al. 2011. Calcineurin controls drug tolerance, hyphal growth, and virulence in Candida dubliniensis. Eukaryot. Cell 10: 803-819.

25. Gupta SS, Ton V, Beaudry V, Rulli S, Cunningham K, Rao R. 2003. Antifungal activity of amiodarone is mediated by disruption of calcium homeostasis. J. Biol. Chem. 278: 28831-28839.

26. Pan J, Hu C, Yu JH. 2018. Lipid biosynthesis as an antifungal target. J. Fungi (Basel) 4: 50.

27. Mahto KK, Singh A, Khandelwal NK, Bhardwaj N, Jha J, Prasad R. 2014. An assessment of growth media enrichment on lipid metabolome and the concurrent phenotypic properties of Candida albicans. PLoS One 9: el13664.

28. Bhattacharya S, Esquivel BD, White TC. 2018. Overexpression or deletion of ergosterol biosynthesis genes alters doubling time, response to stress agents, and drug susceptibility in Saccharomyces cerevisiae. mBio 9: e01291-18.

29. Jorda T, Puig S. 2020. Regulation of ergosterol biosynthesis in Saccharomyces cerevisiae. Genes (Basel) 11: 795.

30. Andres MT, Acosta-Zaldivar M, Fierro JF. 2016. Antifungal mechanism of action of lactoferrin: identification of $\mathrm{H}^{+}$-ATPase (P3AType) as a new apoptotic-cell membrane receptor. Antimicrob. Agents Chemother. 60: 4206-4216.

31. Kjellerup L, Gordon S, Cohrt KO, Brown WD, Fuglsang AT, Winther AL. 2017. Identification of antifungal $\mathrm{H}^{+}$-ATPase inhibitors with effect on plasma membrane potential. Antimicrob. Agents Chemother. 61: e00032-17.

32. Padilla-Lopez S, Pearce DA. 2006. Saccharomyces cerevisiae lacking Btn1p modulate vacuolar ATPase activity to regulate pH imbalance in the vacuole. J. Biol. Chem. 281: 10273-10280.

33. Luna-Tapia A, Peters BM, Eberle KE, Kerns ME, Foster TP, Marrero L, et al. 2015. ERG2 and ERG24 are required for normal vacuolar physiology as well as Candida albicans pathogenicity in a murine model of disseminated but not vaginal Candidiasis. Eukaryot. Cell 14: 1006-1016.

34. Finnigan GC, Ryan M, Stevens TH. 2011. A genome-wide enhancer screen implicates sphingolipid composition in vacuolar ATPase function in Saccharomyces cerevisiae. Genetics 187: 771-783.

35. Chung JH, Lester RL, Dickson RC. 2003. Sphingolipid requirement for generation of a functional v1 component of the vacuolar ATPase. J. Biol. Chem. 278: 28872-28881.

36. Kellermayer R, Aiello DP, Miseta A, Bedwell DM. 2003. Extracellular $\mathrm{Ca}^{2+}$ sensing contributes to excess $\mathrm{Ca}^{2+}$ accumulation and vacuolar fragmentation in a pmr1Delta mutant of S. cerevisiae. J. Cell Sci. 116(Pt 8): 1637-1646.

37. Cui J, Kaandorp JA, Sloot PM, Lloyd CM, Filatov MV. 2009. Calcium homeostasis and signaling in yeast cells and cardiac myocytes. FEMS Yeast Res. 9: 1137-1147.

38. Miller AJ, Vogg G, Sanders D. 1990. Cytosolic calcium homeostasis in fungi: roles of plasma membrane transport and intracellular sequestration of calcium. Proc. Natl. Acad. Sci. USA 87: 9348-9352.

39. Guo Q, Sun S, Yu J, Li Y, Cao L. 2008. Synergistic activity of azoles with amiodarone against clinically resistant Candida albicans tested by chequerboard and time-kill methods. J. Med. Microbiol. 57(Pt 4): 457-462. 